\title{
Arancel externo común y su influencia en el comercio internacional del Paraguay (2000-2019)
}

Common external tariff and its influence on Paraguay's foreign trade (20002019)

\author{
Camila Belén Salcedo Rodríguez ${ }^{1}$ \\ María Antonela Cabral López²
}

\section{Resumen}

A partir del año 1995, se implementó en el Mercado Común del Sur (Mercosur) el Arancel Externo Común (AEC), aunque el mismo se encuentra perforado por distintas excepciones. El objetivo de este trabajo es analizar la influencia del AEC en el comercio internacional del Paraguay durante el periodo 2000-2019. La metodología empleada contempla el diseño documental-bibliográfico, de nivel descriptivo y el enfoque cuantitativo. Por esto, se recurrió al modelo gravitacional del comercio para el estudio propuesto. Entre los principales resultados de esta investigación pueden señalarse que Paraguay posee una economía abierta, con una balanza comercial superavitaria y que el Mercosur es el principal destino de las exportaciones paraguayas y uno de los principales proveedores de sus importaciones. Además, el modelo utilizado permite apreciar que el AEC podría tener una repercusión positiva en el comercio internacional de Paraguay para el periodo de estudio.

Palabras clave: Arancel externo común, Modelo gravitacional, Paraguay

\section{Abstract}

Since 1995, the Common External Tariff (CET) has been implemented in the Southern Common Market (Mercosur), although it is punctuated by several exceptions. The objective of this paper is to analyze the influence of the CET on

Recibido: 30 de septiembre de 2021 Aceptado: 20 de diciembre de 2021 Publicado: 7 de enero de 2022

${ }^{1}$ Economista por la Universidad Nacional de Asunción (UNA), Facultad de Ciencias Económicas, Asunción, Paraguay. Correo electrónico: cambsalcedo@gmail.com ID https://orcid.org/0000-0002-9608-4100

2 Magíster y Candidata a Doctora en Relaciones Internacionales por la Universidad Nacional de La Plata (UNLP). Docente investigadora de la Universidad Nacional de Asunción (UNA), Facultad de Ciencias Económicas, Asunción, Paraguay. Correo electrónico: antocabral@gmail.com ID https://orcid.org/00000003-2074-9767 
Paraguay's international trade during the period 2000-2019. The methodology employed contemplates the documentary-bibliographic design, descriptive level and quantitative approach. Therefore, the gravity model of trade was used for the proposed study. Among the main results of this research is that Paraguay has an open economy, with a surplus trade balance and that Mercosur is the main destination for Paraguayan exports and one of the main suppliers of its imports. In addition, the model used shows that the CET could have a positive impact on Paraguay's international trade during the period under study.

Keywords: Common external tariff, gravitational model, Paraguay

\section{Introducción}

La política exterior como política pública tiene dos características centrales según Busso et. al. (2016): debe tomar en cuenta las necesidades e intereses nacionales y buscar soluciones a nivel internacional, pero simultáneamente resulta afectada por las influencias sistémicas, lo que le da su carácter interméstico ${ }^{3}$. En consideración de lo expuesto, puede apreciarse una vinculación entre los modelos de desarrollo, las estrategias de inserción internacional y su interconexión con la política exterior. Esta autora señala que es posible traducir necesidades internas en alternativas externas, pero que esto contempla una evaluación de las problemáticas, lo que no resulta sencillo.

Como respuesta a la situación gestada durante los años ' 80 del siglo pasado, los que posteriormente fueron conocidos como la "década perdida", los países sudamericanos necesitaban nuevas ideas que otorgasen dinamismo a la región. Con el progresivo regreso de la democracia a los países de la misma, fue posible pensar en nuevas alternativas para el relacionamiento entre países. Una iniciativa muy destacada en este sentido fue la suscripción al Tratado de Asunción (1991), el cual permitió expandir los alcances de los esfuerzos que desde mediados de la década de 1980 emprendieron los presidentes de Argentina y Brasil ${ }^{5}$, y a los que posteriormente se sumaron Uruguay y Paraguay (Granato et al., 2007).

\footnotetext{
${ }^{3}$ Esta expresión, refleja “(...) una combinación tan compleja de elementos internos y externos que han sido denominados "intermésticos" (por el inglés intermestic) en la literatura especializada" (Van Klaveren, 1992). Cabe resaltar los planteos efectuados por Rosenau a finales de la década de 1970 al respecto de este abordaje (Miranda, 2005).

${ }^{4}$ Esta expresión fue acuñada por la CEPAL para reflejar la situación que se experimentaba en la década de 1980 en América Latina en un contexto de alto endeudamiento y la solicitud de ajustes de parte de distintos interlocutores (CEPAL, s. f.).

${ }^{5}$ Previo al nacimiento del Mercosur, Argentina y Brasil suscribieron una serie de documentos que allanaron el camino para la constitución de este esquema de integración. Entre los mismos, cabe destacar: la
} 
El Tratado de Asunción establecía que para el 31 de diciembre de 1994 debía estar conformado un mercado común. El mismo implicaba (Art. 1):

La libre circulación de bienes, servicios y factores productivos entre los países, a través, entre otros, de la eliminación de los derechos aduaneros y restricciones no arancelarias a la circulación de mercaderías y de cualquier otra medida equivalente;

El establecimiento de un arancel externo común y la adopción de una política comercial común con relación a terceros Estados o agrupaciones de Estados y la coordinación de posiciones en foros económicocomerciales regionales e internacionales;

La coordinación de políticas macroeconómicas y sectoriales entre los Estados Partes: de comercio exterior, agrícola, industrial, fiscal, monetaria, cambiaria y de capitales, de servicios, aduanera, de transportes y comunicaciones y otras que se acuerden, a fin de asegurar condiciones adecuadas de competencia entre los Estados Partes;

El compromiso de los Estados Partes de armonizar sus legislaciones en las áreas pertinentes, para lograr el fortalecimiento del proceso de integración.

Es oportuno mencionar también que, además del Tratado de Asunción, el Protocolo de Ouro Preto es clave para este bloque, por medio del cual se asignó personería jurídica al mismo, se estableció la estructura institucional y se consignaron las bases para la unión aduanera. Al respecto de esta última, cabe señalar que se efectuaron una lista de excepciones conforme a las necesidades manifestadas por cada una de las partes y se excluyeron dos sectores para su posterior análisis: la industria automotriz y el azúcar.

Esta investigación tiene como propósito analizar la influencia de la implementación del AEC en el comercio internacional del Paraguay durante el periodo 2000-2019, el cual fue considerado en atención a la disponibilidad de datos existentes, y a que el mismo comprende acontecimientos como la crisis que experimentó el Mercosur a inicios del Tercer Milenio y el superciclo de los commodities, entre otros. El contenido se encuentra distribuido en cinco secciones.

Declaración de Iguazú (1985), el Acta de Integración Argentino-Brasileña (1986); el Tratado de Integración, Cooperación y Desarrollo (1988) y el Acta de Buenos Aires (1990). Posterior a la firma del Tratado de Asunción (1991), el cual dio nacimiento formal al Mercosur, se adoptaron otros instrumentos de relevancia para este bloque, entre los que se encuentran: el Protocolo de Ouro Preto (1994) y el Protocolo de Ushuaia (1998). 
En la primera se presentan los aspectos teóricos que sirven de fundamento al trabajo realizado. En la segunda, por su parte, se detalla la metodología empleada. Seguidamente, en la tercera, se exhibe la evolución de la balanza comercial de Paraguay. Por su parte, en la cuarta se señalan algunas de las barreras al comercio internacional que debe enfrentar el país. Finalmente, se puntualizan los resultados obtenidos a partir del modelo empleado y las conclusiones a las que se arribaron.

\section{Consideraciones teóricas}

Para una cabal comprensión de lo aquí expuesto, se detallarán a continuación algunas de las principales nociones teóricas consideradas en su elaboración. La primera de ellas será el concepto de balanza comercial. La misma es parte de la balanza de pagos y contiene un registro de todas las importaciones y exportaciones de bienes y servicios que ha efectuado un país en un periodo de tiempo determinado. Este concepto es importante debido a que el saldo que presenta indica si el país se encuentra con un déficit o superávit con respecto al comercio con el resto del mundo (Samuelson, 2010).

Un segundo concepto a ser precisado es el de arancel. El mismo es el instrumento principal de la política económica exterior con que cuentan los países, y puede ser definido como aquel impuesto aplicado a la importación de ciertos bienes. Cabe resaltar que tiene la finalidad de incrementar los costos de llevar bienes a un país, de modo de proteger sectores nacionales específicos (Krugman et al., 2012). Un arancel común o regional es aquél que se rige dentro de un espacio económico determinado, generalmente una Unión Aduanera. Se constituye, además, en consideración de las relaciones entre países que suscriben un determinado acuerdo con respecto a mercancías que proceden de terceros países (Asociación Latinoamericana de Integración, s/f).

En cuanto al AEC del Mercosur, Berlinski et al. (2005) presentan de forma muy interesante el camino recorrido. De manera puntual, explican que en Paraguay las reformas en materia comercial se llevaron a cabo a partir de 1989. En ese año, el arancel promedio pasó a ser 14,8\% en lugar del 54\%, manteniéndose relativamente constante hasta 1992. Posteriormente, las reformas lograron reducir el promedio arancelario a $9,5 \%$ en 1995 , por lo que se clasifica a la economía paraguaya como una de las más abiertas en la región. Por esto, es posible aseverar que Paraguay ya contaba con niveles arancelarios moderados antes de la implementación de los acuerdos del Mercosur, a partir de los cuales se observa un incremento del arancel promedio de $9,4 \%$ a $11,1 \%$.

La tendencia importadora del Paraguay, con la implementación de los aranceles concretados en Ouro Preto (1994), se mantuvo inicialmente constante 
debido al mayor intercambio comercial con los demás países miembros, el auge económico en Argentina y Brasil y las excepciones con niveles arancelarios inferiores al AEC. En líneas generales, la implementación del AEC significó para Argentina y Brasil una reducción del promedio de aranceles nominales y de su dispersión. Para Uruguay significó una reducción del arancel promedio, aunque con un incremento de su dispersión, mientras que para Paraguay significó un incremento tanto para su arancel promedio como su dispersión (Berlinski et al., 2005). Cabe señalar también, que a pesar de haberse puesto en vigencia en 1994, el AEC del Mercosur aún se encuentra perforado debido a las listas de excepciones existentes (Gómez Leiva, 2014).

Esto encuentra explicación en que los Estados-parte del bloque siguieron recurriendo a distintos esquemas de excepción, a pesar de los mecanismos de convergencia que se articularon. Asimismo, cabe puntualizar que el uso de mecanismos de excepciones no ha sido homogéneo entre cada uno de los países. Algunos ejemplos de estas perforaciones son: Lista nacional de excepciones (LNE), Régimen de insumos agropecuarios utilizados por Paraguay y Uruguay, Régimen de materias primas utilizados por Paraguay, y Admisión temporaria y draw back, entre otros (Rozemberg et al., 2019).

Una última consideración teórica a ser realizada se vincula con el modelo gravitacional del comercio, el cual será empleado como instrumento teórico para el análisis. El mismo afirma que el flujo de comercio entre dos países se asocia de manera directa con el tamaño de sus economías y de forma indirecta con la distancia existente entre ellos. Además presume la influencia de otras variables, como los tipos de cambios bilaterales, poseer una frontera común, lenguaje común, la cultura, etc.

Sobre el mismo, Krugman et al. (2012) explican su funcionamiento de la siguiente manera: las economías de mayor tamaño tienden a realizar más gastos en importaciones debido a que poseen mucha renta. Asimismo, tienden a captar amplias proporciones del gasto de otros países puesto que producen una extensa gama de mercancías. Con respecto a emplear el modelo gravitacional para evaluar el impacto de los acuerdos comerciales en el comercio internacional, estos autores sostienen que si un acuerdo comercial es eficiente, el mismo debe ocasionar un comercio considerablemente superior entre sus miembros, dados sus niveles de Producto Interno Bruto (PIB) y las distancias entre los mismos.

Por su parte, López \& Muñoz (2008) aseveran que los modelos gravitacionales constituyen una reforma analítica en la economía internacional, con el cual se alcanza a determinar la reacción de emplear instrumentos de política comercial tales como los acuerdos preferenciales, por mencionar un ejemplo. Por 
ello, una gran cantidad de investigaciones lo emplean, entre otras razones, para comprobar el impacto en términos de la desviación y creación de comercio de los distintos acuerdos preferenciales. Usualmente, se emplean variables dummy conforme la participación en los acuerdos. En ellas, un signo positivo en el coeficiente de dicha variable denota creación de comercio y un signo negativo, lo contrario.

Estos mismos autores puntualizan que investigaciones del campo del comercio internacional en América Latina que utilizan el modelo gravitacional consiguieron un alto nivel de ajuste en sus diferentes especificaciones, y evidenciaron la eficiencia del modelo como un instrumento para el análisis comercial en la región (López \& Muñoz, 2008). Como muestra de ello, se mencionan como ejemplos los aportes de Serviss (2003) y Jacobo (2010).

El aporte de la primera de estos autores consiste en afirmar que el Mercosur tuvo un resultado discreto sobre los flujos de comercio bilateral entre sus miembros y que no se aprecian desviaciones de comercio para el período abordado. Por su parte, Jacobo (2010) analiza los determinantes de flujos de comercio entre el Mercosur y la Unión Europea y afirma que los efectos gravitacionales tradicionales entre ambos bloques "son intuitivamente razonables, similar en magnitud a algunos existentes para flujos bilaterales totales de bienes que se comercializan y estadísticamente significativos". Ambos desarrollos sirven para evidenciar la potencialidad que tiene este modelo para analizar el AEC del Mercosur.

\section{Metodología empleada}

El diseño de investigación contempló un trabajo del tipo bibliográfico-documental, de nivel descriptivo y con un enfoque cuantitativo. El periodo considerado en el análisis fue del 2000 al 2019, y para la obtención de datos se recurrió a distintas fuentes, como ser: bases de datos, artículos científicos, informaciones de sitios webs oficiales, libros de texto, tesis e informes de instituciones nacionales e internacionales.

El modelo econométrico utilizado para estimar el impacto del AEC en el comercio internacional es el modelo gravitacional, considerado como una de las herramientas de mayor uso y éxito en el estudio de las corrientes del comercio internacional. Es importante mencionar que para estimar la ecuación se utilizaron técnicas de paneles, es decir, se tomaron de forma conjunta los datos de tipo transversal y datos en serie de tiempo (Cafiero, 2005). 
El modelo gravitacional se estima por el método de Mínimos Cuadrados Generalizados (GLS) ${ }^{6}$ por sus siglas en inglés, con peso de tipo "Cross-Section SUR". Asimismo, los datos empleados son de tipo datos de panel balanceado. La ecuación gravitacional general utilizada (expresado en logaritmos naturales) se especifica de la siguiente manera:

$$
\ln X_{i j t}=\ln \beta_{0}+\beta_{1} \ln Y_{i t}+\beta_{2} \ln Y_{j t}+\beta_{3} \ln D_{i j}+\beta_{4} A E C+\varepsilon
$$

\section{i: Paraguay}

j: Argentina, Brasil, Chile, España, Estados Unidos, India, Italia, Japón, Reino Unido, Rusia, Turquía, Uruguay.

t: 2000, 2001, .., 2019

Donde los subíndices corresponden a los países i y j en el año t, considerando a Paraguay como el país "i" y a los principales socios comerciales como los países “j”. El año "t” se considera desde el año 2000 al 2019.

$\beta_{0}$ : Es el intercepto.

$\mathrm{X}$ : Es el comercio entre los países de estudio, medido por el nivel de exportaciones para cada año en Kilos Netos.

D: Es la distancia en kilómetros entre capitales, de Paraguay a los países seleccionados.

Y: Es el PIB de cada país, medido en dólares Paridad de Poder Adquisitivo (PPA) a precios internacionales constantes de 2011.

AEC: Es una variable dummy, la misma es igual a 1 si los países forman parte del acuerdo regional Mercosur, y disponen del AEC. Por otro lado, toma el valor 0, si los países no forman parte del Mercosur, pero son principales socios comerciales del Paraguay.

$\varepsilon$ : Es la variable estocástica.

Los países seleccionados para desarrollar el modelo fueron 12 de los mayores socios comerciales de Paraguay alrededor del mundo. Entre ellos, tres son los demás socios fundadores del Mercosur (Argentina, Brasil y Uruguay); dos son otros países del continente americano (Chile ${ }^{7}$ y Estados Unidos); cuatro corresponden a Asia (India, Japón, la Federación Rusa y Turquía); y tres son europeos (España, Italia y Reino Unido).

\footnotetext{
${ }^{6}$ Generalized Least Squares (GLS).

${ }^{7}$ Estado asociado del Mercosur.
} 
Los datos del nivel de exportaciones de Paraguay a los países seleccionados fueron obtenidos del Banco Central del Paraguay (BCP, 2020). Por su parte, las variables PIB de cada uno de los países socios y de Paraguay se obtuvieron de la base de datos del Banco Mundial (2020). Por último, los datos de la distancia entre capitales de Paraguay a los países seleccionados se obtuvieron del Centro de Estudios Prospectivos e Información Internacional (CEPII, 2020).

\section{Balanza comercial de Paraguay}

Durante los últimos treinta años, la economía del Paraguay giró en torno a tres pilares: la exportación de commodities agrícolas, la venta de energía eléctrica a los países vecinos, y el comercio de reexportación o de triangulación, el cual está

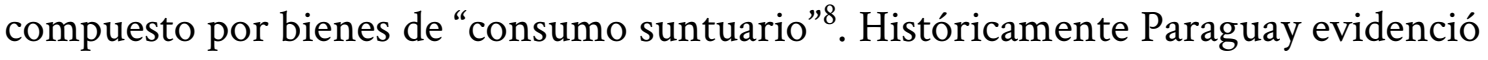
una economía notablemente abierta con relación a los demás países de la región; sin embargo, la ampliación de la oferta exportable y el desarrollo de la industrialización se caracterizaron por ser los principales obstáculos. A la inversa de gran parte de los países latinoamericanos, este país no ha realizado un proceso de industrialización por sustitución de importaciones, lo que resulta en un bajo desarrollo del orden productivo. Pese a eso, goza de relativa estabilidad macroeconómica y bajo endeudamiento público (Rojas, 2015).

La economía paraguaya se caracteriza por tener una política macroeconómica sólida, basada en reglas fiscales y metas de inflación de $4 \%$, con un rango de error de $+/-2 \%$, entre otros rasgos. Asimismo, posee un régimen cambiario flexible que amortigua los shocks externos, baja deuda pública y reservas de divisas equilibradas. No obstante, la concentración de las exportaciones en determinados productos agrícolas influye en el crecimiento y la pobreza, volviéndose vulnerables a las fluctuaciones en los mercados de productos básicos y a los shocks externos (Banco Mundial, 2020).

A partir del Gráfico 1 es posible apreciar la evolución de las exportaciones e importaciones del país. En el mismo se observa que durante todo el periodo 20002019, las exportaciones superaron a las importaciones por un margen de entre el $4 \%$ y el 39\%, siendo el año 2016 el de mayor saldo superavitario. En cuanto al desempeño de las exportaciones, las mayores exportaciones del Paraguay se concretaron en el año 2018, cuando las mismas alcanzaron el monto de USD FOB 13.730,51 millones. En contrapartida, ese mismo año fue el de mayor nivel de importaciones, totalizando un importe de USD FOB 12.917,51 millones.

\footnotetext{
${ }^{8}$ Masi (2006) esboza esta expresión, la que contempla el comercio de bienes tales como: cigarrillos, bebidas alcohólicas, relojes, cámaras fotográficas, juguetes, electrónicos, artículos de informática, etc.
} 
Gráfico 1: Balanza Comercial del Paraguay. Periodo 2000-2019 (en millones de dólares FOB)
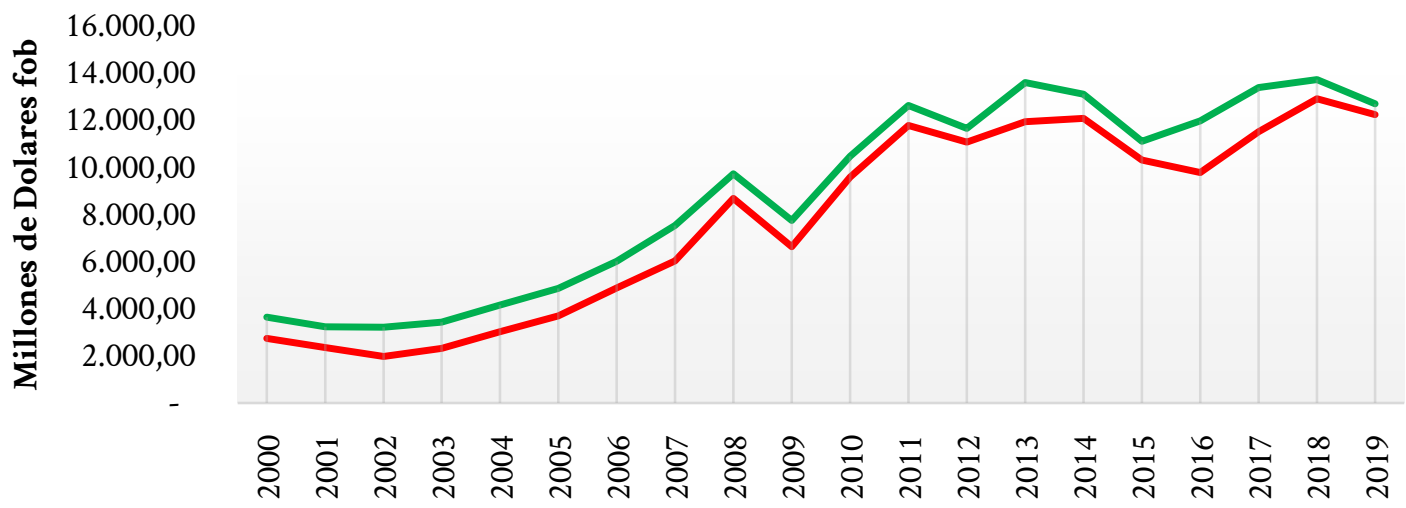
AÑO

Exportaciones totales Importaciones totales

Fuente: Elaboración propia con datos del BCP (2021)

Es oportuno destacar que las exportaciones del país son registradas en tres categorías: las exportaciones totales, las reexportaciones y la categoría "Otros”. La participación de la primera categoría señalada en el total de exportaciones para el periodo de análisis fue de $66,76 \%$ aproximadamente. Por su parte, las reexportaciones representaron el 22,66\%, y la categoría “Otros” constituyó el 10,58\% del total de las exportaciones paraguayas.

Con respecto a los destinos de exportación, pueden evidenciarse dos etapas: 2000-2009 y 2010-2019. En la primera, se observa que Brasil y Argentina han sido los principales destinos de exportación, mientras que Uruguay ocupó el tercer lugar hasta 2006, cediéndoselo después a Chile. Asimismo, es importante mencionar la presencia de Países Bajos, las Islas Caimán, Rusia, Alemania, Suiza, Venezuela e Italia como grandes destinos de las exportaciones paraguayas.

Por otro lado, considerando la segunda etapa, Brasil ha permanecido como principal destino, sin embargo Chile y Rusia desplazaron a Argentina, quien retomó el segundo puesto en 2016. Al igual que en la primera etapa, Alemania, Italia, Países Bajos, Turquía e India fueron importantes destinos de las exportaciones paraguayas. Si se consideran los bloques económicos, a partir de lo planteado precedentemente se observa que el Mercosur es el bloque principal de destino de las exportaciones paraguayas a lo largo del periodo analizado.

Los principales productos que Paraguay exportó durante los años considerados son: la energía eléctrica, la harina y pellets de la extracción del aceite de 
soja, la carne bovina (deshuesada), tanto congelada como fresca, y el aceite de soja en bruto, entre otros.

En lo que respecta al registro de las importaciones, el mismo se realiza en dos categorías: importaciones registradas y otros. La primera de ellas representa alrededor del $91 \%$ de las importaciones totales durante el periodo analizado y la segunda, el $9 \%$.

Con referencia a los orígenes de los productos importados al Paraguay, pueden evidenciarse también dos etapas: 2000-2008 y 2008-2019. En la primera de ellas, las importaciones provinieron principalmente de estos países, en orden de relevancia: Brasil, Argentina, la República Popular China (en adelante China), Estados Unidos y Japón. Sin embargo, dicha situación cambió en la segunda etapa, a partir de la cual se observó que China pasó a liderar el ranking, seguido de Brasil. Por su parte, Argentina y Estados Unidos se han mantenido entre los principales socios comerciales del Paraguay.

A partir de lo expuesto, puede afirmarse que el patrón de comercio de Paraguay es semejante al de los demás países latinoamericanos, que se caracteriza por la exportación de materia prima e importación de manufacturas. Sin embargo, conforme a lo expuesto por Giordano (2004), el Mercosur ha logrado el desarrollo de líneas de producción de superior valor agregado en algunas industrias, pero las mismas son limitadas.

Es oportuno señalar también que la interacción comercial descrita previamente puede ser entendida también a luz de diversos acontecimientos que se registraron durante el periodo de análisis. Al interior del bloque, puede destacarse que Paraguay llegó a ser suspendido del mismo luego del juicio político a Fernando Lugo en 2012 y la puesta en funcionamiento del Fondo para la Convergencia Estructural del Mercosur (FOCEM), el cual brindó financiamiento para importantes proyectos que impulsen la convergencia estructural.

\section{Barreras al comercio internacional de Paraguay}

La política comercial, expresada a través de las medidas arancelarias y no arancelarias, resulta fundamental para el desarrollo del comercio internacional. La estructura arancelaria paraguaya se basa en la Nomenclatura Común del Mercosur (NCM) y el AEC del Mercosur, los cuales a su vez están fundamentados en las normas del Sistema Armonizado de Designación y Codificación de Mercancías, conocido como el Sistema Armonizado (SA) elaborado por la Organización Mundial de Aduanas (OMA). En Paraguay, el Arancel Nacional Vigente (ANV) para el año 2019, contenía unas 10.270 líneas arancelarias a ocho dígitos, con 24 
niveles arancelarios aplicados, que se encuentran entre el 0\% y el 35\% (Subsecretaría de Estado de Economía, s/f).

Con referencia a las Medidas No Arancelarias (MNA), las cuales tienen la potestad de influir en el comercio, repercutiendo tanto en el precio de los bienes comerciados, como en la cantidad o en la calidad de los mismos (Boza \& Fernández, 2014), también son aplicadas en la política comercial de Paraguay. Por su parte, en cuanto a las exportaciones paraguayas, una investigación realizada por Roca (2019) afirma que la gran mayoría de las MNA aplicadas a productos paraguayos son de tipo "Obstáculos técnicos al comercio" y "Medidas sanitarias y fitosanitarias”.

Además de estas medidas, existen otros elementos que pueden influir en el desarrollo del comercio internacional y la aplicación del AEC, que ameritan ser señalados. El primero de ellos se vincula con la situación geográfica del Paraguay. Por no contar con litoral marítimo, el país ha enfrentado históricamente inmensas adversidades para lograr la comunicación con el exterior, las cuales fueron parcialmente contrarrestadas en virtud de las facilidades portuarias y de tránsito que le permitieron sus países vecinos (Moreno Ruffinelli, 2003). No obstante, su localización sigue exponiendo al país a sobrecostos y dificultades para la entrada y salidas de productos.

Un segundo aspecto a considerar es el reconocimiento de la República de China - Taiwán, en adelante Taiwán, por parte de Paraguay. Esta vinculación, que data de 1957, obedecía a las afinidades ideológicas que acercaban a Stroessner y Chiang Kai-shek, al contexto de la Guerra Fría y a la estrategia de política exterior adoptada por Paraguay en aquel momento, pero que permaneció sólida a través del tiempo. En el contexto suscitado por la consolidación de China como uno de los países de mayor peso en el sistema internacional, el debate acerca del reconocimiento a Taipei suma nuevas voces. Si bien es cierto que la cooperación de Taiwán con Paraguay ha trascendido los distintos gobiernos y fue muy importante en distintos campos, los costos que representan para Paraguay esta situación son denominados por Tamayo (2018), quien cita a Urdinez (2018), como el "coste Taiwán”. Conforme a este autor, se refiere a la diferencia entre lo que recibe el Paraguay mediante el apoyo técnico y económico de Taiwán, con lo que no gana en inversiones y apoyo de China ${ }^{9}$.

El reconocimiento de Taiwán por parte de Paraguay ha conducido también a que el Mercosur no pueda negociar un Tratado de Libre Comercio (TLC) con China, uno de los principales socios comerciales de cada uno de los miembros del bloque (Heduvan, 2019). Esto ha sido complejo para los países miembros, ya que

\footnotetext{
${ }^{9}$ Esto amerita un debate más acabado, pero por escapar del alcance de este trabajo, el mismo no es detallado.
} 
este gigante asiático es uno de los principales socios comerciales de cada uno de ellos.

En cuanto a los obstáculos que enfrenta el comercio exterior de Paraguay, cabe señalar un tercer aspecto, vinculado a la reprimarización de la estructura productiva del Mercosur. El hecho de que el comercio intrabloque haya disminuido con el tiempo, y la relevancia de otros socios, como por ejemplo China, haya aumentado, repercute al interior del bloque. Esto se evidencia en la falta de entusiasmo en la constitución de cadenas productivas de mayor sofisticación, la pérdida de interés hacia la integración y el abandono de esfuerzos realizados con este fin, por citar solamente algunas de sus consecuencias, las cuales repercuten en los niveles de comercio que pueden lograrse.

\section{El AEC y el comercio internacional de Paraguay}

En este apartado se expone el análisis de tipo cuantitativo realizado en la presente investigación. A continuación, se presenta nuevamente el modelo considerado (expresado en logaritmos naturales), de manera que facilite la comprensión de los resultados presentados.

$$
\ln X_{i j t}=\ln \beta_{0}+\beta_{1} \ln Y_{i t}+\beta_{2} \ln Y_{j t}+\beta_{3} \ln D_{i j}+\beta_{4} A E C+\varepsilon
$$

$\mathrm{i}=$ Paraguay

$\mathrm{j}=$ Argentina, Brasil, Chile, España, Estados Unidos, India, Italia, Japón,

Reino Unido, Rusia, Turquía, Uruguay.

$\mathrm{t}=2000,2001, \ldots, 2019$

$\beta_{0}$ : Es el intercepto

$\mathrm{X}$ : Es el comercio entre los países de estudio, medido por el nivel de exportaciones

D: Es la distancia en kilómetros entre las capitales de los países seleccionados.

Y: Es el PIB de cada país

AEC: Es una variable dicotómica o dummy

$\varepsilon$ : Es la variable estocástica

En primer término, se detalla el tratamiento que tuvieron los datos de los países. Para la realización de la estimación se otorgó un identificador (ID) a cada uno de ellos, los cuales se presentan en la Tabla 1. Asimismo, cada uno de ellos está detallado junto con la distribución de la variable dicotómica o dummy, la cual toma el valor 1 para los socios comerciales del Paraguay que integran el Mercosur. 
Tabla 1: Distribución de la variable dicotómica para los países seleccionados en el modelo gravitacional

\begin{tabular}{|c|c|c|}
\hline ID & PAÍS & AEC \\
$\mathbf{1}$ & Argentina & 1 \\
\hline $\mathbf{2}$ & Brasil & 1 \\
\hline $\mathbf{3}$ & Uruguay & 1 \\
\hline $\mathbf{4}$ & Chile & 1 \\
\hline $\mathbf{5}$ & USA & 0 \\
\hline $\mathbf{6}$ & Japón & 0 \\
\hline $\mathbf{7}$ & Reino Unido & 0 \\
\hline $\mathbf{8}$ & Rusia & 0 \\
\hline $\mathbf{9}$ & India & 0 \\
\hline $\mathbf{1 0}$ & Turquía & 0 \\
\hline $\mathbf{1 1}$ & Italia & 0 \\
\hline $\mathbf{1 2}$ & España & 0 \\
\hline
\end{tabular}

Fuente: Elaboración propia

En segunda instancia es preciso efectuar una revisión de los signos esperados de cada uno de los coeficientes de las variables que son utilizadas en el modelo. En cuanto al PIB, se estima que el signo debería ser positivo. Considerando la distancia entre los países y que la misma se relacionaba de forma directa con los costos de transporte y los de transacción, sería esperable que el signo sea negativo en dicha variable. Finalmente, se esperaría que la variable dummy AEC demuestre un signo positivo puesto que, de acuerdo al estudio de la Fundación INAI (2011), la función principal del AEC es lograr una política arancelaria coordinada para los productos frente a terceros países, el cual favorezca al comercio en la región a costa de las importaciones procedentes de países externos al bloque.

En la Tabla 2 se observan los principales resultados de la regresión del modelo gravitacional que se ha utilizado para estimar la influencia del AEC en el comercio internacional del Paraguay. Con dichos resultados se puede afirmar que los coeficientes de las variables incluidas tienen los signos esperados, es decir, el modelo sí evidencia la relación directa entre el comercio y el tamaño de la economía, la relación inversa con la distancia entre el Paraguay y los países seleccionados, y el signo positivo de la variable dummy de AEC muestra el impacto positivo de la implementación del AEC del Mercosur en el comercio internacional del Paraguay. 
Tabla 2: Principales resultados obtenidos del modelo gravitacional

\begin{tabular}{|l|r|r|r|c|}
\hline \multicolumn{1}{|c|}{ Variable } & Coeficiente & Error Estándar & Estadístico t & P >t \\
\hline C & $-2,86324$ & 0,939352 & $-3,048101$ & 0,0026 \\
\hline LOG(Yi*Yj) & 0,581446 & 0,020876 & 27,85295 & 0,0000 \\
\hline LOG(D) & $-1,114259$ & 0,119996 & $-9,285773$ & 0,0000 \\
\hline AEC & 1,176035 & 0,184365 & 6,37886 & 0,0000 \\
\hline
\end{tabular}

Fuente: Elaboración propia.

Además de los signos de los coeficientes estimados para cada una de las variables, es preciso analizar otros valores arrojados por el modelo. En primer término, se considera el valor del coeficiente de determinación conocido como " $\mathrm{R}$ cuadrado". Sus valores pueden oscilar entre 0 y 1 , en el que un valor de 1 significa un ajuste perfecto del modelo y, por otro lado, un valor de 0 infiere que no hay relación entre la variable regresada y la regresora (Gujarati \& Porter, 2010). En este caso, el mismo alcanza un 0,90 , con lo cual se puede concluir que la proporción de variación total en la variable dependiente es explicada de forma satisfactoria por las variables independientes.

Tabla 3: Principales estadísticos obtenidos del modelo gravitacional

\begin{tabular}{|l|l|}
\hline Estadístico & Resultado \\
\hline Total de observaciones de datos de panel (balanceadas) & 240 \\
\hline Periodo de tiempo & 20 \\
\hline Datos de Corte Transversal incluidos & 12 \\
\hline R-cuadrado & 0,901799 \\
\hline R-cuadrado ajustado & 0,900551 \\
\hline Estadístico F & 722,4099 \\
\hline Prob $>$ F & 0 \\
\hline Estadístico Durbin-Watson & 1,631709 \\
\hline
\end{tabular}

Fuente: Elaboración propia

El programa utilizado también proporciona el estadístico $t$ de student y su probabilidad asociada ( $\mathrm{p}$-valor), en el cual al observar que el p-valor es cero, nos indica que se rechaza la hipótesis de que las medias son iguales, por lo que las variables consideradas son estadísticamente significativas (Guerrero \& Gómez, 
2017). A su vez, se observa que la estimación en conjunto demuestra un estadístico $\mathrm{F}$ significativo, lo que se evidencia por el p-valor menor al $5 \%$.

Los resultados de la regresión estimada son de utilidad para evidenciar que los supuestos del modelo gravitacional se cumplen. A la vez, los mismos también permiten apreciar que para el caso analizado, puede considerarse positiva la influencia de la adopción del AEC en el comercio internacional de Paraguay. No obstante, es importante mencionar que el impacto del AEC fue considerado a través de un instrumento, el modelo gravitacional, y de forma agregada, por lo que se estima que sería de mucha relevancia efectuar nuevos análisis a través de otras herramientas, y que se lleven a cabo estudios sectoriales que posibiliten realizar un análisis más acabado sobre el tema.

\section{Conclusiones}

La investigación efectuada permitió apreciar distintos matices en lo que respecta al comercio internacional de Paraguay. En primer término, se pudo observar que la balanza comercial paraguaya ha presentado superávit durante todo el periodo analizado. A través del mismo, tanto las exportaciones como las importaciones se han incrementado progresivamente, y el Mercosur como bloque resultó clave para ambas actividades. Pese a ello, el patrón de comercio de Paraguay se sigue caracterizando por la exportación de materia prima e importación de manufacturas.

Otra de las aristas a tenerse en cuenta en lo que respecta al comercio internacional, se vincula con aquellos elementos que componen la política comercial. Por esta razón se revisó la estructura arancelaria, las medidas no arancelarias y otros factores, como ser la localización y el relacionamiento internacional con otros países. Fruto de este análisis pudo apreciarse que la economía paraguaya es abierta y que se encuentra expuesta a distintos factores exógenos.

Un tercer aspecto a ser señalado es la influencia del AEC. Por medio de la aplicación del modelo gravitacional pudo verificarse que los coeficientes de las variables analizadas tienen los signos esperados y son estadísticamente significativos. Por esto, se puede aseverar que, como afirma la literatura, el flujo de comercio internacional medido por las exportaciones es directamente proporcional al tamaño de la economía medido por el PIB, e inversamente proporcional a la distancia entre los mismos. Además, la variable dummy de AEC presenta un signo 
positivo, lo que da un indicio de que repercutió de manera positiva en el comercio internacional de Paraguay durante el periodo 2000-2019.

Cabe destacar que dicho efecto fue estimado de acuerdo a un instrumento determinado: el modelo gravitacional. No obstante, se debe tener en cuenta que existen diversas herramientas que podrían estimar el tipo de repercusión que el AEC podría tener en el comercio internacional del Paraguay, por lo que se considera pertinente recurrir a ellas para obtener un resultado más robusto. Asimismo, se recomienda la realización de estudios sectoriales, para visualizar de forma diferenciada el impacto específico de los aranceles aplicados a los distintos sectores productivos.

Es oportuno mencionar que los hallazgos de esta investigación son interesantes en el marco de los debates en torno a cuál debería ser el futuro del bloque. A 30 años de la firma del Tratado de Asunción, son diferentes las voces que mencionan la necesidad de "repensar" cuál es el camino que el bloque debe tomar, y el hecho de ser una zona de libre comercio o una unión aduanera es uno de los interrogantes principales que se plantean. Por ello, se estima que el estudio del AEC, y su relación con el comercio exterior de cada uno de los países es de relevancia para generar insumos que colaboren en las distintas instancias de análisis.

Finalmente, es importante resaltar que para la implementación de una política económica exterior efectiva y acorde a las necesidades de cada estado, es necesario efectuar un análisis amplio que integre las dimensiones externa e interna, así como el aspecto político y económico.

\section{Referencias}

Asociación Latinoamericana de Integración (ALADI). (s/f). Arancel Externo Común [Sitio web oficial]. Glosario de Términos Aduaneros y de Comercio Exterior. http://www.aladi.org

Banco Central del Paraguay (BCP). (2020). Sistema Integrado de Comercio Exterior (SICEX) [Base de datos].

Banco Central del Paraguay (BCP). (2021). Anexo Estadístico del Informe Económico [Base de datos].

Banco Mundial. (2020). Indicadores del Desarrollo Mundial-DataBank [Base de datos]. Grupo Banco Mundial. Disponible en: https://databank.bancomundial.org

Banco Mundial. (2020). Paraguay: Panorama general [Sitio web oficial]. World Bank. https://www.bancomundial.org 
Berlinski, J., Kume, H., Vaillant, M., Miranda, P., Ons, A., \& Romero, C. (2005). Aranceles a las importaciones en el MERCOSUR: El camino al Arancel externo Común. Documento de Trabajo/FCS-DE; 8/05.

Boza, S., \& Fernández, F. (2014). Chile Frente a la Regulación sobre Medidas no Arancelarias de la Organización Mundial del Comercio. Estudios internacionales (Santiago), 46(178), 65-82.

Busso, A. (Ed.). (2016). Modelos de desarrollo e inserción internacional. UNR Editora. https://rephip.unr.edu.ar

Cafiero, J. A. (2005). Modelos Gravitacionales para el Análisis del Comercio Exterior. Revista del CEI(Comercio Exterior e Integración), 13.

Centro de Estudios Prospectivos e Información Internacional (CEPII). (2020). GeoDist. http://www.cepii.fr/

Comisión Económica para América Latina y el Caribe (CEPAL). (s.f.). Información histórica - Evolución de las ideas de la CEPAL. Recuperado de: https://www.cepal.org/sites/default/files/pages/files/informacion_historica.p df

Congreso de la Nación Paraguaya. (1991). Ley no 9/91 que aprueba y ratifica el tratado para la constitución de un mercado común (Tratado de Asunción). 131 Asunción, Paraguay. Disponible en: https://www.mre.gov.py

Fundación INAI. (2011). La importancia del Arancel Externo Común del Mercosur a 20 años de la conformación del bloque. Revista de la Bolsa de Comercio de Rosario. http://www.bcr.com.ar

Giordano, P. (2004). Mirando al MERCOSUR y al mundo: Estrategia de comercio e integración para Paraguay (Vol. 27). Bid-intal.

Gómez Leiva, E. (2014). El Arancel Externo Común del MERCOSUR como incentivo a la competitividad de sus miembros.

Granato, L., Oddone, C. N., \& Simón, M. P. (2007). Política, economía y energía frente al siglo veintiuno: La integración regional y el fortalecimiento de la democracia en Paraguay. Población y Desarrollo, 33, 64-79.

Guerrero, H. A., \& Gómez, E. (2017). Guía para el análisis estadístico básico, pruebas de hipótesis y regresiones en el EViews.

Gujarati, D. N., \& Porter, D. C. (2010). Econometría (5a ed.). McGraw-Hill Interamericana.

Heduvan, J. (2019). La relación Paraguay-Taiwán, un ejemplo de éxito como bastión de resistencia regional. 
Jacobo, A. D. (2010). Una estimación de una ecuación gravitacional para los flujos bilaterales de manufacturas Mercosur-Unión Europea. Economia Aplicada, 14(1), 67-79.

Krugman, P. R., Obstfeld, M., \& Melitz, M. J. (2012). Económica internacional teoría y politica / International Economics Theory and Politics (9a ed.). Pearson Educación.

López, D., \& Muñoz, F. A. (2008). Los modelos de gravedad en América Latina: El caso de Chile y México. Comercio exterior, 58(11), 803-813.

Miranda, R. (2005). La política exterior argentina como objeto de estudio: Un enfoque, algunas pistas. https://repositoriosdigitales.mincyt.gob.ar/vufind/Record/SEDICI_9d885cf8 3891650d51a72c4350e194d5

Moreno Ruffinelli, J. A. (2003). La política exterior del Paraguay. Revista Mexicana de politica exterior, 69, 169-181.

Roca, L. (2019). Paraguay: informe sobre las medidas no arancelarias en la región para determinados productos. Asociación Latinoamericana de Integración (ALADI).

Rojas, D. C. C. (2015). Integración Productiva Paraguay-Brasil: Nuevos Pasos en el Relacionamiento Bilateral. Fronteiras e Relações Internacionais, Curitiba, Editora Ithala.

Rozemberg, R., Campos, R., Gayá, R., Makuc, A., \& Svarzman, G. (2019). Informe Mercosur: hacia un cambio necesario. Banco Interamericano de Desarrollo. Recuperado https://publications.iadb.org/publications/spanish/document/Informe_MER COSUR_tiempos_de_cambio_es_es.pdf

Samuelson, P. A. (2010). Economía: Con aplicaciones a Latinoamérica (19a ed.). McGraw-Hill.

Serviss, L. (2003). Los efectos de los arreglos comerciales regionales: Análisis para el MERCOSUR. Universidad de Sevilla. http://www.asepelt.org

Subsecretaría de Estado de Economía. (s/f). Aranceles a la Importación [Sitio web oficial]. https://www.economia.gov.py

Tamayo, E. (2018). Paraguay, repensando la política exterior. Novapolis, 13, 141162.

Van Klaveren, A. (1992). Entendiendo las políticas exteriores latinoamericanas: modelo para armar. Estudios Internacionales, 25(98), 169-216. 